\title{
Validación de la escala Seguridad en el Sistema Familiar (SIFS) en dos muestras españolas de adolescentes y jóvenes residentes con su familia e institucionalizados*
}

Validating the Security in the Family System Scale (SIFS) in two Samples of Spanish Adolescents and Youth Living with their Families and Institutionalized

Recibido: 16 de marzo de 2014 | Aceptado: 16 de marzo de 2016

\author{
Silvia López-LARROSA ** \\ PAUla MENDIRI-RUIZ-DE-AlDA *** \\ VANESA SÁNCHEZ SOUTO **** \\ Universidade da Coruña, Galicia, España
}

doi: 10.11144/Javeriana.upsy15-2.vessf

Para citar este artículo: López-Larrosa, S., MendiriRuiz-de-Alda, P., \& Sánchez Souto, V. (2016). Validación de la escala Seguridad en el Sistema Familiar (SIFS) en dos muestras españolas de adolescentes y jóvenes residentes con su familia e institucionalizados. Universitas Psychologica, 15(2), 361-370. http:// dx.doi.org/10.11144/Javeriana.upsy15-2.vessf

Artículo de investigación. No tuvo financiación por parte de ninguna entidad. Agradecimientos: gracias a Josu Mujica Lizaso por su inestimable ayuda con los análisis estadísticos.

** Doctora en Psicología desde 1993. Profesora titular de Universidad en el Departamento de Psicología Evolutiva y de la Educación. Correo electrónico: silvia.lopez.larrosa@udc.es

**** Doctora desde 2000. Profesora titular en el Departamento de Filosofía y Métodos de Investigación en Educación. Correo electrónico: paula.mendiri@ udc.es

****** Doctora desde 2016 en el Departamento de Psicología Evolutiva y de la Educación. Correo electrónico: tecvss01@udc.es

\section{RES U MEN}

En este trabajo, se analizaron las propiedades psicométricas de la escala de Seguridad en el Sistema Familiar (SIFS) (Forman \& Davies, 2005) que mide la percepción de seguridad o amenaza que tienen los hijos acerca del sistema familiar. El punto de partida teórico de la SIFS es la teoría de la seguridad emocional (Cummings \& Davies, 2010). La muestra estuvo compuesta por un total de 845 adolescentes y jóvenes españoles, de los cuales 180 estaban institucionalizados (media de edad 15.67 años) y 665 vivían con sus familias (media de edad 14.9). Se realizaron análisis factoriales confirmatorios y análisis de invarianza configural, métrica, escalar y estricta. Los resultados indicaron que el instrumento es adecuado para evaluar la seguridad en el sistema familiar en muestras de adolescentes y jóvenes españoles institucionalizados y no institucionalizados, identificándose las tres dimensiones que predice el modelo (seguridad, preocupación y desvinculación) con una buena consistencia interna y puntuaciones comparables.

Palabras clave:

seguridad emocional; familia; adolescentes; protección de menores

\section{A B S T R A C T}

The psychometric properties of the Security in the Family System scale (SIFS) (Davies \& Forman, 2005) are analyzed in this paper. SIFS measures children's perceived security in the family system grounded on Emotional Security theory (Cummings \& Davies, 2010). Particpants were 845 Spanish adolescents and youth, 180 of whom were institutionalized (mean age 15.67 years) while 665 lived with their families (mean age 14.9 years). Confirmatory factor analyses and configural, metric,scalar and strict invariance analyses were performed. Results showed that the instrument is adequate to evaluate Spanish adolescents' and youth's security in the family whether they live with their families or they are institutionalized. The three dimensions predicted by the theory were identified (security, preoccupation and disengagement) with a good internal consistency and comparable punctuations.

Keywords

Emotional security; family; adolescents; child protection 
El impacto del conflicto marital sobre los hijos se ha abordado desde distintas perspectivas teóricas, entre las cuales destaca la teoría de la seguridad emocional (EST, por el acrónimo inglés) (Cummings \& Davies, 2010; Cummings, Schermerhorn, Davies, Goeke-Morey, \& Cummings, 2006; Davies, Harold, Goeke-Morey, \& Cummings, 2002; López-Larrosa, Sánchez Souto, \& Mendiri-Ruiz-de-Alda, 2012). Según la EST, los hijos tienen como meta principal el sentirse protegidos en su familia (Cummings \& Davies, 2010; Davies et al., 2002). La seguridad emocional se alcanza cuando los lazos familiares son sólidos y estables, y los miembros de la familia responden y están emocionalmente disponibles para los hijos aun cuando se produzcan situaciones de estrés (Cummings et al., 2006). Una situación potencialmente generadora de estrés es el conflicto entre los padres, aunque no todos los conflictos entre los padres producen un efecto estresante en los hijos. Por esto, Cummings y Davies (2010) distinguen dos tipos de conflicto marital: el conflicto constructivo y el destructivo.

Los elementos distintivos del conflicto constructivo son el apoyo, la discusión tranquila, la solución de problemas y el afecto positivo; mientras que los del conflicto destructivo son estar a la defensiva, la agresión física, la amenaza, la persecución, la retirada, la hostilidad verbal (insulto personal) y no verbal, así como una emocionalidad negativa (Cummings, Goeke-Morey, \& Papp, 2003; GoekeMorey, Cummings, Harold, \& Shelton, 2003).

Según la EST, la amenaza que supone el conflicto destructivo incrementa la preocupación del hijo sobre su seguridad, aumentando, por tanto, su reactividad al conflicto entre sus padres. Esto implica que la exposición reiterada al conflicto destructivo entre los padres hace a los hijos más sensibles al conflicto en lugar de insensibilizarlos. Por otro lado, se ha comprobado que el conflicto constructivo no supone una amenaza para la seguridad emocional de los hijos (McCoy, Cummings, \& Davies, 2009).

Aunque durante un tiempo, los efectos del conflicto sobre los niños o adolescentes se estudiaron preguntando especialmente a los padres (Grych, Seid, \& Ficham, 1992) o a otros adultos relacionados con ellos como sus profesores (CoO- per, Holman, \& Braithwaite, 1983), también los investigadores se han planteado que es esencial preguntar o estudiar a los hijos directamente (Emery \& O'Leary, 1982; Justicia \& Cantón, 2011). Las técnicas pueden ser variadas: cuestionarios, autoinformes, entrevistas, medidas observacionales (Easterbrooks, Cummings, \& Emde, 1994; Emery \& O'Leary, 1982; Jacobson, 1978; Jouriles, Pfiffner, \& O'Leary, 1988; López-Larrosa, Escudero, \& Cummings, 2009; Schaefer, 1965) o incluso, medidas fisiológicas como la tasa cardíaca, la conductancia de la piel o los niveles de corticoides en la orina (Davies, Sturge-Apple, Cicchetti, \& Cummings, 2007, 2008; El-Sheikh \& Harger, 2001; El-Sheikh, Keller, \& Erath, 2007). En línea con las propuestas que defienden el estudio del impacto del conflicto contando con los hijos como primeros informantes, se desarrolló la Security in the Family System Scale (SIFS), que tiene como base conceptual la teoría de la seguridad emocional (Forman $\&$ Davies, 2005). Dado que la EST es aplicable a todo el sistema familiar (Cummings et al., 2006), era y es necesario considerar un instrumento de evaluación de la seguridad emocional de los hijos en el sistema familiar, esto es, ir más allá de la seguridad que proporciona la observación y valoración de la solidez de la relación entre los padres. SIFS plantea la importancia de realizar una evaluación global de la seguridad percibida en el sistema familiar "que no puede obtenerse mediante el estudio de las interacciones diádicas específicas o la suma de las mismas" (Forman \& Davies, 2005, p. 901). Teniendo en cuenta la carencia de instrumentos para evaluar la percepción que los hijos tienen de todo el sistema familiar y sus implicaciones para su bienestar, Forman y Davies (2005) concibieron la SIFS como un instrumento para evaluar la confianza que un niño o adolescente tiene en su familia como base de protección y apoyo, especialmente durante las situaciones de estrés.

Con la SIFS se trata de identificar las tres estrategias que los hijos desarrollan para la preservación de su seguridad emocional: segura, preocupada y de rechazo o desvinculación. La estrategia segura se da cuando los hijos confían en sus figuras de socialización familiares como fuentes de seguridad, apoyo 
y predictibilidad. Las dos estrategias inseguras son preocupación y desvinculación. La estrategia de preocupación consiste en estar intranquilo por la familia. La estrategia de desvinculación consiste en alejarse de la familia. Cuando hay conflictos destructivos y estos se van prolongando en el tiempo, en general los hijos usan estrategias inseguras, bien amplificando la importancia de los conflictos, por tanto, estando preocupados, para estar preparados e identificar pronto cualquier amenaza a su bienestar; bien, rechazando y desvinculándose de su familia para disminuir la importancia que esta tiene en sus vidas. En los análisis realizados a la escala SIFS, en su versión inglesa, se extrajeron tres factores que se corresponden exactamente con estas tres estrategias: preocupación, seguridad y desvinculación (Forman \& Davies, 2005).

Los autores inciden en la importancia de aplicar y validar la SIFS con muestras de edades más amplias que la de los 12 a 15 años que estudiaron; además, consideran necesario incluir otros grupos culturales y, se podría añadir, otras circunstancias vitales. Habitualmente, la seguridad emocional se ha estudiado con muestras de chicos que viven con dos progenitores (Cummings et al., 2006; Davies, Woitach, Winter, \& Cummings, 2008). No obstante, algunos adolescentes no viven con sus padres $\mathrm{u}$ otros adultos que puedan actuar como tales. Se trata de la población de menores institucionalizados o en acogimiento residencial. En concreto, en la zona de España donde se realizó este estudio, Galicia, el número total de menores en acogimiento residencial durante el año 2011 fue de 617, de los cuales 528 tenían las edades más aproximadas a los participantes en este estudio (Ministerio de Sanidad, Servicios sociales e Igualdad, 2013).

La población de menores institucionalizados no vive con sus familias porque estas no ofrecen las garantías de cuidado, no pueden hacerse cargo de ellos o porque los hijos han realizado conductas sancionables, de modo que las autoridades competentes se hacen cargo de ellos. La institucionalización se produce mientras no se alcanza la mayoría de edad (18 años), pero en ocasiones, por decisión de las autoridades, se prolonga. La institucionalización se concibe como una situación transitoria porque o bien se produce adopción o acogimiento o bien la familia mejora y se produce la integración familiar. De haberse realizado conductas legalmente sancionables, el regreso a la familia se producirá una vez cumplidas las condiciones impuestas legalmente.

Los modelos unicausales en el ámbito de la conducta antisocial han sido sustituidos por modelos multicausales que tienen en cuenta factores de riesgo y protección en diversos ámbitos además del familiar: la escuela, la comunidad y las variables individuales y de los iguales son también relevantes. En una investigación con una muestra de 2440 adolescentes y jóvenes de la región geográfica de los participantes en este estudio, el conflicto familiar era una de las variables relacionadas con la conducta antisocial, junto con otras variables (López-Larrosa \& Rodríguez-Arias Palomo, 2012). Por tanto, en la población institucionalizada por haber realizado conductas antisociales, el conflicto aparece como factor de riesgo. Según Martín y Dávila (2008), además del conflicto familiar, que en un $16.86 \%$ de los casos está próximo a la violencia, otros factores de riesgo presentes en los menores infractores son las pautas de crianza inadecuadas con un estilo parental permisivo-indulgente, maltrato infantil (un $18.53 \%$ lo ha sufrido) y falta de afecto, teniendo solo vínculo de apego con uno de sus progenitores (Luzón García \& Domínguez Alonso, 2014). Por su parte, según los mismos autores, un $77.5 \%$ de los jóvenes en acogimiento residencial ingresaron en el centro por inadecuado cumplimiento de las funciones parentales y el resto, $22.5 \%$, debido al imposible cumplimiento. Estudios meta-analíticos han puesto de relieve que los vínculos de apego inseguro y desorganizado son los más predominantes entre los niños y adolescentes que viven en las instituciones (Lionetti, Pastore, \& Barone, 2015; Zaccagnino, Cussino, Preziosa, Veglia, \& Carassa, 2015).

Dado que la SIFS permite evaluar cómo los adolescentes y jóvenes perciben su sistema familiar con respecto a su propia seguridad emocional, lo cual, según hemos visto, va más allá del vínculo de apego con un progenitor, y dado que este estudio no se ha hecho con muestra española institucionalizada y no 
institucionalizada, se tradujo la escala al castellano. Para su validación en ambas muestras, se amplió la edad de los participantes con respecto al estudio norteamericano original. Puesto que uno de los objetivos de la institucionalización es que el menor pueda retornar a su medio familiar, contar con un instrumento que nos permita evaluar su seguridad emocional favorecerá la toma de decisiones a la hora de determinar si la familia reúne unas condiciones adecuadas para la reintegración familiar.

\section{Método}

\section{Participantes}

El número total de participantes fue de 845 adolescentes y jóvenes que cursaban Educación Secundaria Obligatoria o postobligatoria (bachiller y formación profesional) en diferentes centros educativos públicos rurales y urbanos de la región noroeste de España (Galicia).

Un total de 180 de estos adolescentes y jóvenes vivían en una de las 17 instituciones participantes. Estas instituciones son centros donde residen los menores bajo el sistema de protección de la región autonómica donde se recogieron los datos. El resto de la muestra, 665 chicos y chicas, sí vivían con sus familias y acudían a uno de los 7 institutos en los que se recogieron los datos. La Tabla 1 presenta los datos sociodemográficos de ambas muestras.

\section{Instrumento}

Security in the Family System Scale (SIFS) (Forman \& Davies, 2005): evalúa la percepción de los hijos de seguridad en su familia. Puede ser administrada a partir de los 10 años. Consta de 24 ítems con cinco alternativas de respuesta, desde muy en desacuerdo (1) a muy de acuerdo (5). El cuestionario fue traducido por dos psicólogos expertos que trabajaron independientemente y después revisado por un nativo inglés experto en traducción. Se respetaron los criterios de traducción inversa al tiempo que se buscó un equilibrio con el significado cultural de los ítems (Muñiz, Elosua, \& Hambleton, 2013).

En la versión inglesa, se extrajeron tres factores: preocupación (p. ej., "tengo la sensación de que mi familia tendrá muchos cambios que yo no espero"), seguridad (p. ej., "creo que los miembros de mi familia estarán cerca para ayudarme en el futuro") y desvinculación (p. ej., "cuando pasa algo malo en mi familia, me gustaría vivir en una familia diferente"). Para aceptar esta solución de tres factores, se eliminaron dos ítems.

La escala de 22 ítems ha demostrado una adecuada consistencia interna y fiabilidad test-retest, así como validez y capacidad predictiva. La consistencia interna y la fiabilidad test-retest de la subescala de preocupación es de 0.88-0.85, los valores para la subescala de seguridad son de 0.85-0.82 y para la subescala de desvinculación son de 0.85 0.85. La SIFS ha sido utilizada en muestras ameri-

\section{TABLA 1}

Datos sociodemográficos de los participantes

\begin{tabular}{lll}
\hline & Participantes que viven con su familia & Participantes que viven en centros \\
Media edad (DE) & $14.9(1.79)$ & $15.67(1.74)$ \\
\% Mujeres $(n)$ & $53(353)$ & $40.8(73)$ \\
\% Hombres $(n)$ & $47(312)$ & $59.2(107)$ \\
Familia de procedencia & $\%(n)$ & $\%(n)$ \\
Familia nuclear & $81.67(543)$ & $34.31(62)$ \\
Familia monoparental & $14.87(99)$ & $39.05(70)$ \\
Madre única responsable & $12.6(84)$ & $30.17(54)$ \\
Padre único responsable & $2.25(14.96)$ & $15.39(27.7)$ \\
Otras formas familiares & $3.45(23)$ & $21.89(39)$ \\
\hline
\end{tabular}

Fuente: elaboración propia. 
canas y también en otros países como Irán (Gharehbaghy, Aguilart-Vafaie, \& Allahyary, 2009). En un estudio previo con 500 adolescentes españoles (López-Larrosa et al., 2012), hubo que eliminar tres ítems con bajo peso en la escala y esta quedó reducida a 19 ítems.

\section{Procedimiento}

Para poder acceder a los centros de menores, se solicitó permiso a la autoridad autonómica competente. Una vez conseguido, se contactó con los centros y se solicitó el permiso de sus directores para recoger los datos. Tras la respuesta afirmativa de éstos, los chicos y chicas fueron informados sobre el estudio y dieron su asentimiento.

Para acceder a la muestra de adolescentes y jóvenes que vivían con sus familias, se contactó con los institutos y se solicitó el permiso del director para aplicar los cuestionarios. El director consultó a los docentes y estos informaron a los alumnos para pedir el consentimiento de los padres. Una vez obtenidos estos consentimientos y el asentimiento de los chicos, se procedió a aplicar los cuestionarios.

La aplicación de los cuestionarios en los centros de menores y en los institutos siguió un procedimiento similar: se hizo grupalmente en el tiempo en que los alumnos estaban con su tutor o algún docente que expresamente hubiese indicado que permitía la aplicación de los cuestionarios. En todos los casos estuvo presente una de las investigadoras de este trabajo. Los alumnos debían contestar anónimamente.

\section{Análisis de datos}

Se estimó la consistencia interna por medio del coeficiente alpha de Cronbach. Se analizó la estructura factorial mediante el análisis factorial confirmatorio (CFA). La equivalencia entre el grupo institucionalizado y no institucionalizado fue evaluada a través de un estudio progresivo de invarianza, en el que se examinaron modelos cada vez más rigurosos a través de la adición de restricciones sobre la matriz de patrones factoriales (invarianza configural), las cargas factoriales (invarianza métrica), los intercep- tos (invarianza escalar) y las varianzas residuales (invarianza estricta).

Los análisis factoriales se efectuaron con el paquete lavaan (Rosseel, 2012) en el entorno R (R Development Core Team, 2015). El método de estimación para los análisis factoriales confirmatorios fue el de máxima verosimilitud. La evaluación del ajuste de los modelos a los datos se realizó con base en el índice de bondad de ajuste (CFI; Hu \& Bentler, 1999) y la raíz media cuadrática del error de aproximación (RMSEA). Se consideraron aceptables aquellos modelos con valores iguales o superiores a 0.9 en CFI (Bentler, 1990; Tabachnick \& Fidell, 2005) e iguales o menores a 0.08 en RMSEA (Browne \& Cudeck, 1993). Como criterio de aceptación de los modelos métrico, escalar y estricto de invarianza, se requirió que la diferencia en CFI entre dos modelos inmediatos fuera igual o inferior a 0.01 .

\section{Resultados}

En la Tabla 2 se presentan los resultados descriptivos de los ítems y la consistencia interna de los factores Preocupación, Desvinculación y Seguridad. El porcentaje de varianza explicada por las tres subescalas es de $52.68 \%$.

\section{CFA con tres factores correlacionados}

El modelo de tres factores correlacionados fue aceptado. Desde la aproximación lineal, los índices de bondad de ajuste se situaron entre 0.9 y 0.95 , y el error cuadrático medio de aproximación fue siempre inferior a 0.08 (Tabla 3).

\section{Análisis progresivo de la invarianza factorial}

La evaluación progresiva de la invarianza comenzó por el modelo de invarianza configural. Los índices de ajuste obtenidos permitieron aceptar la equivalencia de los modelos de medida básicos entre los dos grupos tratando las variables como continuas. Los índices de bondad de ajuste fueron CFI $=0.936$ y la raíz media cuadrática no fue superior a 0.08 , RMSEA $=0.054$. 
TABLA 2

Medias y desviaciones estándar de los items, y alpha de Cronbach de las subescalas

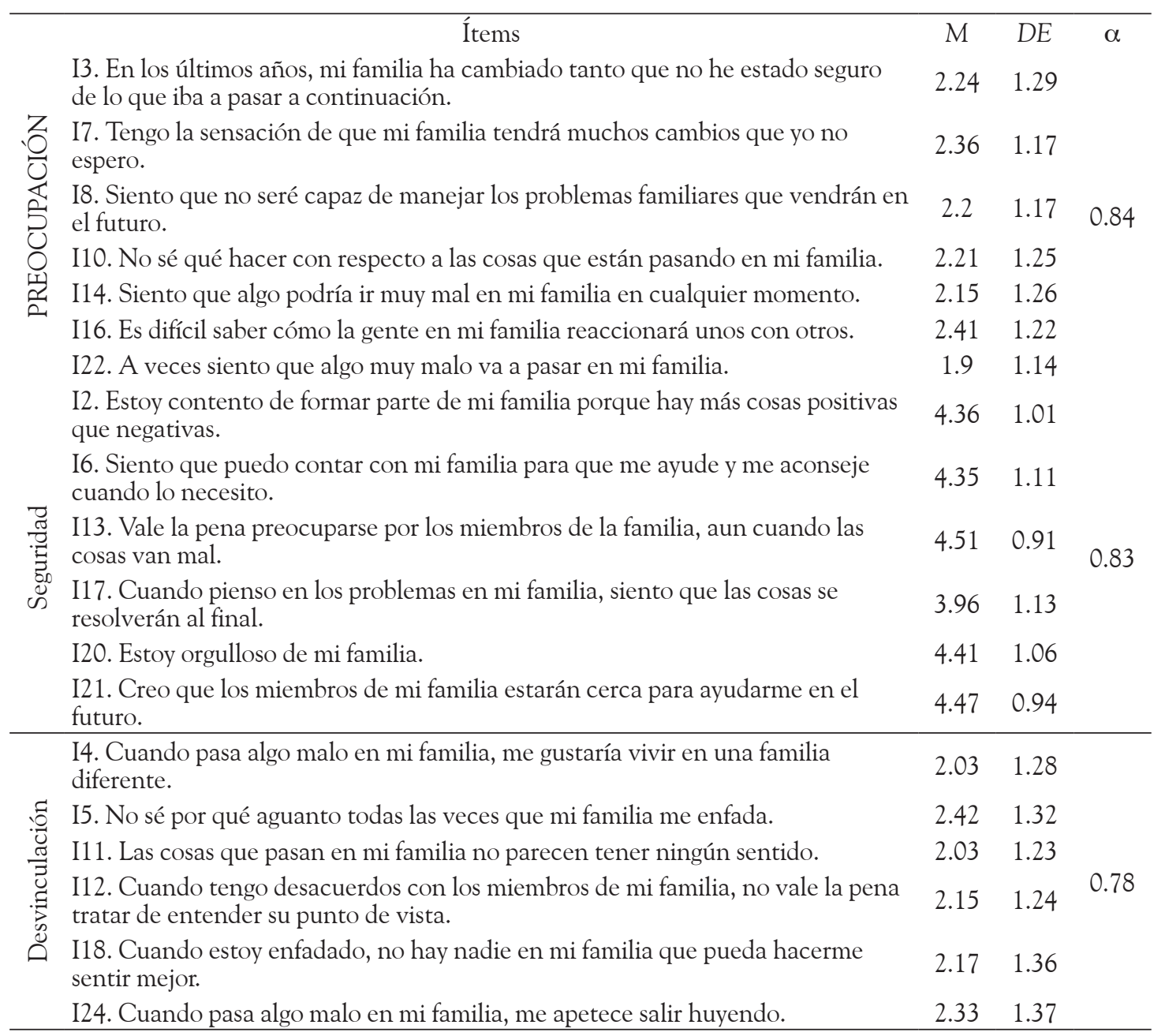

Fuente: elaboración propia.

TABLA 3

Análisis factorial confirmatorio

\begin{tabular}{ccccc}
\hline Grupo & $g l$ & $\chi^{2}$ & CFI & RMSEA \\
\hline & & Variables continuas & \\
Total & 149 & 476.419 & 0.942 & 0.053 \\
1 & 149 & 402.761 & 0.939 & 0.052 \\
2 & 149 & 239.798 & 0.924 & 0.06 \\
\hline
\end{tabular}

Nota. El grupo 1 son los chicos que viven con sus familias y el grupo 2 son los chicos que viven en centros.

Fuente: elaboración propia.

La invarianza métrica se caracterizó añadiendo al modelo base restricciones sobre las cargas factoriales. Los valores recogidos en la Tabla 4 permiten aceptar este nivel de invarianza. La diferencia entre el valor del modelo base y el de invarianza métrica en CFI fue inferior a $0.01, \Delta \mathrm{CFI}=0.005$.

El modelo de anidamiento en este punto del proceso nos llevó a evaluar la equivalencia entre los 
Validación de la escala Seguridad en el Sistema Familiar (SIFS) en dos muestras ESPAÑOLAS DE ADOLESCENTES Y JÓVENES RESIDENTES CON SU FAMILIA E INSTITUCIONALIZADOS

TABLA 4

Análisis factorial confirmatorio multigrupo

\begin{tabular}{cccccccccc}
\hline \multirow{2}{*}{ Modelo } & $\begin{array}{c}\text { Parámetro } \\
\text { liberado }\end{array}$ & $g l$ & $\chi^{2}$ & CFI & RMSEA & $\Delta g l$ & $\Delta \chi^{2}$ & $\Delta$ CFI & $\Delta$ RMSEA \\
IC & & 298 & 642.559 & 0.936 & 0.054 & & & & \\
IM & & 314 & 683.535 & 0.931 & 0.055 & 16 & 40.975 & 0.005 & 0 \\
IESC & & 330 & 722.547 & 0.927 & 0.055 & 16 & 39.013 & 0.004 & 0 \\
IEST & & 349 & 964.122 & 0.885 & 0.067 & 19 & 241.575 & 0.041 & 0.012 \\
& $\Theta_{I 2}$ & 348 & 879.499 & 0.901 & 0.062 & 18 & 156.952 & 0.026 & 0.007 \\
& $\theta_{\mathrm{I} 18}$ & 347 & 855.319 & 0.905 & 0.061 & 17 & 132.772 & 0.022 & 0.006 \\
& $\Theta_{\mathrm{I} 10}$ & 346 & 834.351 & 0.909 & 0.06 & 16 & 111.804 & 0.018 & 0.005 \\
& $\theta_{\mathrm{I} 14}$ & 345 & 820.555 & 0.911 & 0.059 & 15 & 98.008 & 0.015 & 0.004 \\
& $\theta_{\mathrm{I} 16}$ & 344 & 809.793 & 0.913 & 0.059 & 14 & 87.246 & 0.014 & 0.004 \\
& $\theta_{\mathrm{II} 2}$ & 343 & 799.373 & 0.915 & 0.058 & 13 & 76.826 & 0.012 & 0.003 \\
& $\Theta_{\mathrm{I} 22}$ & 342 & 789.599 & 0.917 & 0.058 & 12 & 67.052 & 0.01 & 0.003 \\
\hline
\end{tabular}

Nota. $\mathrm{IC}=$ Invarianza configural, IM = Invarianza métrica, IESC = Invarianza escalar, IEST = Invarianza estricta.

Fuente: elaboración propia.

TABLA 5

Parámetros en el modelo final

\begin{tabular}{|c|c|c|c|c|c|c|}
\hline \multirow{2}{*}{ Ítem } & \multicolumn{3}{|c|}{ Carga } & \multirow{2}{*}{ Intercepto } & \multicolumn{2}{|c|}{ Varianza residual } \\
\hline & Preocupación & Seguridad & Desvinculación & & Grupo 1 & Grupo 2 \\
\hline I3 & 0.876 & & & 2.113 & 0.929 & 0.929 \\
\hline I7 & 0.752 & & & 2.228 & 0.802 & 0.802 \\
\hline I8 & 0.69 & & & 2.068 & 0.884 & 0.884 \\
\hline $\mathrm{I} 10$ & 0.874 & & & 2.048 & 0.693 & 1.229 \\
\hline $\mathrm{I} 14$ & 1 & & & 1.963 & 0.503 & 0.887 \\
\hline $\mathrm{I} 16$ & 0.57 & & & 2.329 & 1.065 & 1.590 \\
\hline $\mathrm{I} 22$ & 0.873 & & & 1.759 & 0.493 & 0.774 \\
\hline $\mathrm{I} 2$ & & 0.768 & & 1.511 & 0.343 & 1.089 \\
\hline I6 & & 0.766 & & 1.568 & 0.774 & 0.774 \\
\hline $\mathrm{I} 13$ & & 0.534 & & 1.431 & 0.586 & 0.586 \\
\hline $\mathrm{I} 17$ & & 0.689 & & 1.967 & 0.871 & 0.871 \\
\hline $\mathrm{I} 20$ & & 1 & & 1.466 & 0.273 & 0.273 \\
\hline $\mathrm{I} 21$ & & 0.812 & & 1.437 & 0.314 & 0.314 \\
\hline $\mathrm{I} 4$ & & & 0.928 & 1.912 & 0.958 & 0.958 \\
\hline $\mathrm{I} 5$ & & & 0.904 & 2.296 & 1.08 & 1.08 \\
\hline $\mathrm{I} 11$ & & & 0.923 & 1.903 & 0.823 & 0.823 \\
\hline $\mathrm{I} 12$ & & & 0.709 & 2.056 & 1.044 & 1.563 \\
\hline $\mathrm{I} 18$ & & & 0.864 & 2.016 & 1.069 & 1.987 \\
\hline $\mathrm{I} 24$ & & & 1 & 2.216 & 1.099 & 1.099 \\
\hline
\end{tabular}

Nota. En negrita ítems y parámetros liberados. El grupo 1 son los chicos que viven con sus familias y el grupo 2 son los chicos que viven en centros.

Fuente: elaboración propia. 
valores interceptales, modelo de invarianza escalar. Los índices mostraron un buen ajuste de este modelo. La diferencia entre los índices de bondad de ajuste fue de 0.004 . Aceptada la invarianza escalar, concluimos la equivalencia entre los parámetros interceptales; los dos modelos evaluados fueron equivalentes respecto a las cargas factoriales y los interceptos.

Por último, se evaluó el grado más alto de invarianza, la invarianza estricta. Los valores mostrados en la tabla inducen a rechazar el modelo, $\Delta \mathrm{CFI}=$ 0.041. El estudio detallado de los ítems permitió concluir la no invarianza de la varianza residual del ítem 2, de manera que se volvió a testar el modelo estricto, liberando la restricción sobre dicho parámetro. Aunque los valores en el índice de bondad de ajuste y en la raíz media cuadrática del modelo de invarianza estricta parcial, CFI $=0.901$ y RMSEA $=0.062$, fueron sustancialmente mejores que en el modelo anterior, $\mathrm{CFI}=0.885$ y $\mathrm{RMSEA}=0.067$, la diferencia en CFI respecto al modelo de invarianza escalar continuó siendo inaceptable $(\Delta \mathrm{CFI}=0.026)$. El mismo proceso se repitió sucesivamente hasta dar con un modelo aceptable; lo que llevó a liberar las restricciones sobre la igualdad de varianzas en los ítems 2, 10, 12, 14, 16, 18 y 22. El modelo parcialmente invariante aceptado bajo el criterio $\Delta \mathrm{CFI} \leq 0.01$ arrojó un índice de bondad de ajuste $\mathrm{CFI}=0.917$, y su raíz media cuadrática fue $R M S E A=0.058$. Las varianzas residuales de los siete ítems sobre los que se relajaron las restricciones fueron sistemáticamente mayores en el grupo 2, esto es, los chicos que vivían en centros de menores (Tabla 5).

\section{Discusión}

La escala en su versión castellana identifica los tres factores del instrumento original que predice la teoría de la seguridad emocional: Seguridad, Preocupación y Desvinculación. En este estudio, los tres factores obtuvieron unos niveles de consistencia interna similares a los obtenidos en la versión original y, además, los índices de bondad de ajuste son aceptables.

Aunque Preocupación y Desvinculación son estrategias que denotan inseguridad emocional, se ha optado por considerarlas independientemente, puesto que, desde el punto de vista de la teoría, aunque estadísticamente estén relacionadas positivamente, son conceptualmente diferentes. La preocupación implica que los hijos están inquietos por su familia en el momento actual y en el futuro. Por su parte, a través de la desvinculación, el hijo se mantiene al margen de la familia, por ejemplo, deseando pertenecer a una familia diferente o sintiendo que no vale la pena entender el punto de vista de los demás miembros de la familia.

Dado que se pretendía evaluar la equivalencia de un modelo de medida en la muestra de adolescentes que viven con su familia y la muestra de los que residen en centros, se analizó progresivamente la invarianza factorial. El CFA permitió validar el modelo analizado satisfactoriamente en los dos grupos y en la totalidad de la muestra. Se alcanzó el nivel de equivalencia escalar entre los chicos que viven en centros y los que viven con sus familias. El modelo de invarianza estricta parcial solo pudo ser aceptado tras estimar libremente las varianzas residuales de siete de los 19 ítems que componen SIFS. En la escala Preocupación se liberaron las restricciones sobre los ítems 10, 14, 16 y 22; en la escala Seguridad sobre el ítem 2 y en Desvinculación sobre los ítems 12 y 18.

Según el análisis progresivo de invarianza, esta versión de SIFS puede utilizarse para evaluar los mismos aspectos psicológicos en ambas muestras y las puntuaciones son comparables, si bien debe considerarse que la composición de las puntuaciones observadas no es la misma, siendo mayor la variabilidad residual de algunos ítems en la población institucionalizada.

En conclusión, SIFS, también en su versión española, se encuadra entre los escasos instrumentos de evaluación de los procesos familiares para su uso en investigación y como instrumento de screening para identificar tanto población adolescente emocionalmente segura como insegura. Además, puede emplearse con adolescentes de edades superiores a las utilizadas en el estudio original y con población que vive tanto con su familia como en centros. No obstante, coincidimos con Davies, Winter y 
Cicchetti (2006) en que se debe ser muy cauto a la hora de utilizar la teoría o, como en este caso, los resultados de SIFS para proponer intervenciones. Por ejemplo, si se observa que un adolescente tiene una desvinculación elevada, se podría suponer que esto es lo que hay que trabajar. Pero, sin una intervención paralela o conjunta con su familia que garantice que esta es más segura, reducir su desvinculación puede ser perjudicial.

\section{Referencias}

Bentler P. M. (1990). Comparative fit indexes in structural models. Psychological Bulletin, 107, 238-246. doi:10.1037/0033-2909.107.2.238

Browne, M. W., \& Cudeck, R. (1993). Alternate ways of assessing model fit. En K. A. Bollen \& J. S. Long (Eds.), Testing structural equation models (pp. 136162). Newbury Park, CA: Sage.

Cooper, J. E., Holman, J., \& Braithwaite, V. A. (1983). Self-esteem and family cohesion: The child's perspective and adjustment. Journal of Marriage and Family, 45(1), 145-159. doi:10.2307/351303

Cummings, E. M., \& Davies P. T. (2010). Marital conflict and children. An emotional security perspective. Nueva York: Guildford Press.

Cummings, E. M., Goeke-Morey, M. C., \& Papp, L. (2003). Children's responses to everyday marital conflict tactics in the home. Child Development, 74(6), 1918-1929.

Cummings, E. M., Schermerhorn, A. C., Davies, P. T., Goeke-Morey, M. C., \& Cummings, J. S. (2006). Interparental discord and child adjustment: Prospective investigations of emotional security as an explanatory mechanism. Child Development, 77(1), 132-152.

Davies, P. T., Harold, G. T., Goeke-Morey, M. C., \& Cummings, E. M. (2002). Child emotional security and interparental conflict. Monographs of the Society for Research in Child Development, 270, 67(3), 1-114.

Davies, P. T., Sturge-Apple, M. L., Cicchetti, D., \& Cummings, E. M. (2007). The role of child adrenocortical functioning in pathways between interparental conflict and child maladjustment. Developmental Psychology, 43(4), 918-930.
Davies, P. T., Sturge-Apple, M. L., Cicchetti, D., \& Cummings, E. M. (2008). Adrenocortical underpinnings of children psychological reactivity to interparental conflict. Child Development, 79(6), 1693-1706.

Davies, P. T., Winter, M. A., \& Cicchetti, D. (2006). The implications of emotional security theory for understanding and treating childhood psychopathology. Development and Psychopathology, 18(3), 707-735.

Davies, P. T., Woitach, M. J., Winter, M. A., \& Cummings, E. M. (2008). Children's insecure representations of the interparental relationship and their school adjustment: The mediating role of attention difficulties. Child Development, 79(5), 1570-1582.

Easterbrooks, M. A., Cummings, E. M., \& Emde, R. N. (1994). Young children's responses to constructive marital disputes. Journal of Family Psychology, 8(2), 160-169.

El-Sheikh, M., \& Harger, J. (2001). Appraisals of marital conflict and children's adjustment, health and psychological reactivity. Developmental Psychology, 37(6), 875-885.

El-Sheikh, M., Keller, P., \& Erath, S. A. (2007). Marital conflict and risk for child maladjustment over time: Skin conductance level reactivity as a vulnerability factor. Journal of Abnormal Child Psychology, 35(5), 715-727.

Emery, R., \& O'Leary, K. (1982). Children's perceptions of marital discord and behavior problems of boys and girls. Journal of Abnormal Child Psychology, 10(1), 11-24.

Forman, E., \& Davies, P. T. (2005). Assessing children's appraisals of security in the family system: The development of the Security in the Family System (SIFS) Scales. Journal of Child Psychology and Psychiatry, 46(8), 900-916.

Gharehbaghy, F., Aguilar-Vafaie, M., \& Allahyary, A. A. (2009). Children's appraisal of interparental conflict and psychopathological symptoms: An investigation of mediating and moderating mechanisms. Iranian Journal of Psychiatry and Clinical Psychology, 15(1), 70-80.

Goeke-Morey, M. C., Cummings, E. M., Harold, G. T., \& Shelton, K. H. (2003). Categories and continua of destructive and constructive marital conflict 
tactics from the perspective of U.S. and Welsh children. Journal of Family Psychology, 17(3), 327-338.

Grych, J. H., Seid, M., \& Fincham, F. D. (1992). Assessing marital conflict from the child's perspective: The children's perception of interparental conflict scale. Child Development, 63(3), 558-572.

Hu, L., \& Bentler, P. M. (1999). Cut-off criteria for fit indexes in covariance structure analysis: Conventional criteria versus new alternatives. Structural Equation Modeling, 6(1), 1-55.

Jacobson, D. S. (1978). The impact of marital separation/divorce on children. II Interparent hostility and child adjustment. Journal of Divorce, 2(1), 3-19.

Jouriles, E. N., Pfiffner, L. J., \& O'Leary, S. G. (1988). Marital conflict, parenting and toddler conduct problems. Journal of Abnormal Child Psychology, 16(2), 197-206.

Justicia, M. J., \& Cantón, J. (2011). Conflictos entre padres y conducta agresiva y delictiva en los hijos. Psicothema, 23(1), 20-25.

Lionetti, F., Pastore, M., \& Barone, L. (2015). Attachment in institutionalized children: A review and meta-analysis. Child Abuse EF Neglect, 42, 135-145.

López-Larrosa, S., Escudero, V., \& Cummings, E. M. (2009). Preschool children and marital conflict: A constructive view. European Journal of Developmental Psychology, 6(2), 170-179.

López-Larrosa, S., \& Rodríguez-Arias Palomo, J. L. (2012). Factores de riesgo y de protección en el consumo de drogas y la conducta antisocial de adolescentes y jóvenes españoles. International Journal of Psychological Research, 5, 5-13.

López-Larrosa, S., Sánchez Souto, V., \& Mendiri-Ruiz-de-Alda, P. (2012). Los adolescentes y el conflicto interparental destructivo: impacto en la percepción del sistema familiar y diferencias según el tipo de familia, la edad y el sexo de los adolescentes. Universitas Psychologica, 11(4), 1262-1274.

Luzón García, A., \& Domínguez Alonso, J. (2014). El menor infractor que comete su primer delito. Revista sobre la Infancia y la Adolescencia, 7, 40-57. doi: 10.4995/reinad.2014.3336

Martín, E., \& Dávila, L. M. (2008). Redes de apoyo social y adaptación de los menores en acogimiento residencial. Psicothema, 20(2), 229-235.

McCoy, K., Cummings, E. M., \& Davies, P. T. (2009). Constructive and destructive marital conflict, emotional security and children's prosocial behavior. Journal of Child Psychology and Psychiatry, 50(3), 270-279.

Ministerio de Sanidad, Servicios Sociales e Igualdad. (2013). Boletín de datos estadísticos de medidas de protección a la infancia (datos 2011). Madrid: Dirección General de Servicios para la familia y la infancia.

Muñiz, J., Elosua, P., \& Hambleton, R. K. (2013). Directrices para la traducción y adaptación de los test (2.a ed.). Psicothema, 25(2), 151-157.

R Development Core Team. (2015). R: A Language and Environment for Statistical Computing. Viena, Austria: R Foundation for Statistical Computing.

Rosseel, Y. (2012). lavaan: An R Package for Structural Equation Modeling. Journal of Statistical Software, 48(2), 1-36. http://dx.doi.org/10.18637/jss.v048.i02

Schaefer, E. S. (1965). Children's reports of parental behavior: An inventory. Child Development, 36(2), 413-424.

Tabachnick, B. G., \& Fidell, L. S. (2005). Using multivariate statistics (5.a ed.). Boston, MA: Pearson.

Zaccagnino, M., Cussino, M., Preziosa, A., Veglia, F., \& Carassa, A. (2015). Attachment representation in institutionalized children: A preliminary study using the child attachment interview. Clinical Psychology and Psychotherapy, 22(2), 165-175. 